\title{
Exact results for BCS systems
}

\author{
Xi-Wen Guan*†, Angela Foerster ${ }^{\dagger}$, Jon Links ${ }^{\dagger \dagger}$ and Huan-Qiang Zhou ${ }^{\dagger \dagger}$ \\ ${ }^{\dagger}$ IF-UFRGS, Porto Alegre, Brazil, Bento Gonçalves Avenue, 9500 \\ ${ }^{\dagger}$ Centre for Mathematical Physics, School of Physical Sciences, \\ The University of Queensland, 4072, Australia \\ E-mail: guan@if.ufrgs.bri
}

\begin{abstract}
In this paper, we discuss the construction of integrable BCS models in the framework of the quantum inverse scattering method. Specifically, the reduced BCS model, which is based on the $s u(2)$ algebra, a model of two coupled BCS systems, based on the $s u(4)$ algebra and an extended pairing model associated to the $u(2 \mid 2)$ symmetry are presented.
\end{abstract}

\section{Introduction}

The Bethe ansatz method is one of the most powerful techniques for treating one dimensional quantum systems. It was first introduced by Bethe in 1931 to solve the isotropic Heisenberg model [i] was developed further by Baxter, who solved the XYZ-Heisenberg model [2]

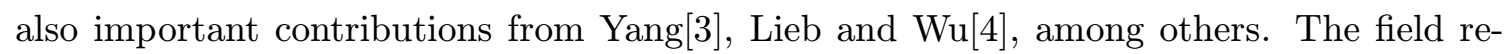
ceived a great impulse with its algebraic formulation proposed by Faddeev, Takhtajan and

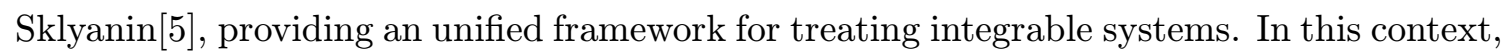
it is important to mention that Bethe ansatz methods received also considerable interest from the condensed matter community due to its application in the exact solution of some

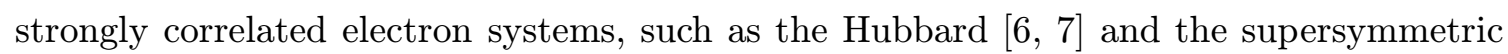

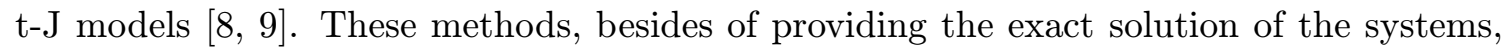
allow for the computation of relevant physical quantities.

Recently, the study of exact solutions has attracted renewed interest due to its application in the theory of metallic ultra-small grains. In particular, recent experiments of single-electron tunneling spectroscopy on ultra-small AL grains performed by Ralph, Black and Tinkham [i] $[1]$ in have shown that it is not valid to apply the BCS mean field theory for

\footnotetext{
${ }^{*}$ Speaker.
} 
systems of nanoscale size. Consequently, the search for exact solutions have increased considerably and a large amount of theoretical investigations on the reduced BCS Hamiltonian [i] 1,i] describing electron pairing correlations in the canonical ensemble have been performed.

It is by now well established that this model is exactly solvable [i] 1 in] has an underlying $s u(2)$ symmetry and is integrable [1]

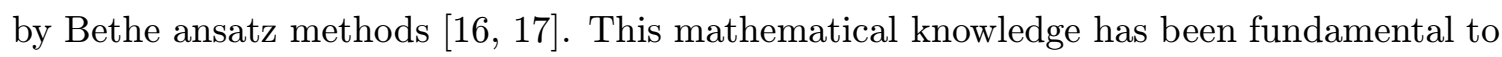
establish and improve physical concepts of the standard BCS theory of superconductivity, such as the nature of the pairing correlations, the limitation of the mean-field approaches for ultra-small systems, among others (for an excellent review see $\left.{ }_{1}^{1} \overline{1} \bar{y}_{-1}\right)$. Moreover, it allows for an easier computation of relevant quantities, such as finite-temperature properties, form

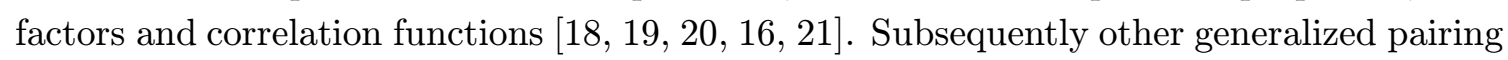
Hamiltonians have been constructed in order to gain an insight into the physical properties

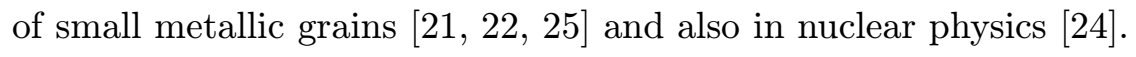

Such generalized models can be interpreted as BCS models of pairing correlations, at least in the sense that every simple Lie algebra can be generated by a system of simple roots

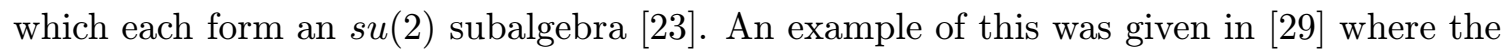
Lie algebra employed was $s u(4)$. In this instance, the Hamiltonian constructed takes the form of two BCS systems which individually describe pairing interactions for the protons and neutrons and the scattering of bound proton pairs-neutron pairs. In this paper we discuss the construction of integrable BCS models in the framework of the algebraic Bethe ansatz method. Specifically, the reduced BCS model, which is based on the $s u(2)$ algebra, a model of two coupled BCS systems based on the $s u(4)$ algebra and and extended pairing model associated to the superalgebra $u(2 \mid 2)$ are presented.

The paper is organized as follows. In section 2 , we present the construction and prove the integrability of the standard $s u(2)$ BCS model. In section $\overline{3}_{1,}$, we present the construction of two coupled BCS systems through the algebraic Bethe ansatz and discuss its exact solution. In section i, we introduce an extended BCS model associated with the Lie superalgebra $u(2 \mid 2)$. A summary of the main results can be found in section

\section{The reduced BCS Hamiltonian}

Let's us start by presenting the Hamiltonian for the reduced BCS model,

$$
H_{\mathrm{BCS}}=\sum_{j, \sigma= \pm}^{\Omega} \epsilon_{j \sigma} c_{j, \sigma}^{\dagger} c_{j, \sigma}-g \sum_{j, k}^{\Omega} c_{j,+}^{\dagger} c_{j,-}^{\dagger} c_{k,-} c_{k,+}
$$

which consists of a kinetic term and an interaction term describing the attraction between Cooper pairs in time reverse states. Above $c_{j \sigma}^{\dagger}, c_{j \sigma}$ are the creation and annihilation operators in time-reversed states $\mid j, \pm>$ with energies $\epsilon_{j}, g$ is the BCS pairing constant. The sums are taken over a set of $\Omega$ doubly degenerate energy levels $\epsilon_{j}$. The total number

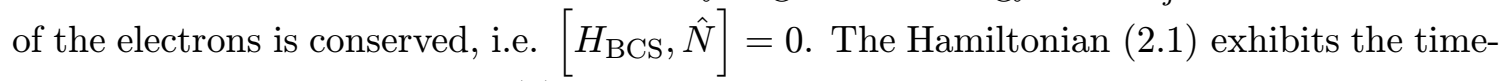
reversed symmetry and the $s u(2)$ symmetry as well as the blocking effect-singly-occupied levels do not participate in the pair scattering. It is meant that every unpaired electron 
Pauli-blocks the scattering of other pairs into its own singly-occupied level. It is remarkable that the spectrum of the model reveals parity effect. In an even- $N$ grain, all excited states involve at least one broken Cooper pair, hence lie a gap $\Delta E$ above the fully-paired BCS ground state. In an odd- $N$ grain all states have at least one unpaired electron, hence no significant gap exists between ground- and excited-states.

Let us review now how this model can be constructed from the algebraic Bethe ansatz method. It is shown that the Hamiltonian $\left(\overline{2} . \overline{1} \overline{1}_{i}\right)$ can be obtained from the $s u(2)$ spin model via a realization

$$
H_{\mathrm{BCS}}=\sum_{j} \epsilon_{j} b_{j}^{+} b_{j}-g \sum_{j, k} b_{j}^{+} b_{k} .
$$

Above $b_{j}(a), b_{j}^{+}(a)$ are annihilation and creation operators for the hard-core bosons with the commutation relations

$$
\begin{aligned}
& {\left[b_{j}, b_{k}^{+}\right]=\delta_{j, k}\left(1-2 \hat{N}_{j}\right),} \\
& \hat{N}_{j}=b_{j}^{+} b_{j}, \quad\left(b^{+}\right)^{2}=0 .
\end{aligned}
$$

In order to built up a mechanism to construct an integrable pairing model, let us first recall the quantum $R$-matrix associated with the Lie algebra $s u(2)$, which acts in the tensor product of two 2-dimensional spaces $V \otimes V$ and can be written as

$$
R(\lambda)=\frac{(\lambda . I \otimes I+\eta P)}{(\lambda+\eta)} .
$$

Above $\lambda$ is the usual spectral parameter, $P$ is the permutation operator with matrix elements $P_{\alpha \beta, \gamma \delta}=\delta_{\alpha \delta} \delta_{\beta \gamma}, \quad \alpha, \beta, \gamma, \delta=1,2$ and $\eta$ is the quasiclassical limit parameter; i.e.

$$
\lim _{\eta \rightarrow 0} R(\lambda)=I \otimes I
$$

It is known that this $R$-matrix satisfies the Yang-Baxter equation (YBE)

$$
R_{12}(\lambda-\mu) R_{13}(\lambda) R_{23}(\mu)=R_{23}(\mu) R_{13}(\lambda) R_{12}(\lambda-\mu) .
$$

The R-matrix may be viewed as the structural constants for the Yang-Baxter algebra generated by the monodromy matrix $T(\lambda)$, namely,

$$
R_{12}(\lambda-\mu) \stackrel{1}{T}(\lambda) \stackrel{2}{T}(\mu) \stackrel{2}{T}(\mu) \stackrel{1}{T}(\lambda) R_{12}(\lambda-\mu) .
$$

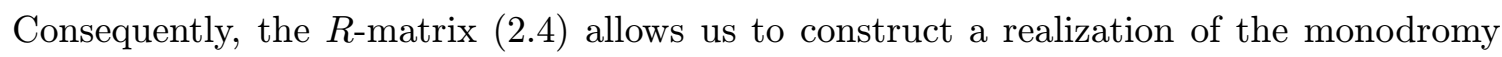
matrix through

$$
T(\lambda)=G_{0} R_{0 \Omega}\left(\lambda-\epsilon_{\Omega}\right) \cdots G_{0} R_{01}\left(\lambda-\epsilon_{1}\right) .
$$

Here the subscript 0 denotes the auxiliary space and $G$ satisfying

$$
[R, G \otimes G]=0
$$

is a class of c-valued solutions of the YBE (2.25). As a consequence of the Yang-Baxter algebra $\left(\overline{2} . \overline{6}^{\prime}\right)$, the transfer matrices $t(\lambda)=\operatorname{tr}_{0} \mathrm{~T}(\lambda)$ mutually commute for different values 
of the spectral parameter $\lambda$. This transfer matrix is the starting point in the construction of a $s u(2)$-type Gaudin Hamiltonian, from which we can obtain the $s u(2)$ pairing Hamiltonian, as will be shown below. For this purpose we make the following identification for the basis states

$$
|1\rangle=|0\rangle,|2\rangle=b^{+}|0\rangle
$$

and choose the G-matrix to be given by $G_{0}=\exp \left(\frac{\eta}{g \Omega} \hat{N}_{0}\right)$ to construct the transfer matrix $t(\lambda)$. It can be verified that

$$
\begin{aligned}
t\left(\epsilon_{j}\right)= & \operatorname{tr}_{0}\left\{G_{0} R_{0 \Omega}\left(\epsilon_{j}-\epsilon_{\Omega}\right) \cdots G_{0} P_{0 j} \cdots G_{0} R_{01}\left(\epsilon_{j}-\epsilon_{1}\right)\right\} \\
= & G_{j} R_{j, j-1}\left(\epsilon_{j}-\epsilon_{j-1}\right) \cdots G_{j} R_{j 1}\left(\epsilon_{j}-\epsilon_{1}\right) G_{j} R_{j, \Omega}\left(\epsilon_{j}-\Omega\right) \\
& \cdots G_{j} R_{j, j+1}\left(\epsilon_{j}-\epsilon_{j+1}\right) G_{j} .
\end{aligned}
$$

Above $\operatorname{tr}_{0} P_{0 j}=1$. Next, taking the quasiclassical limit, we find

$$
\begin{aligned}
\left.R_{j, k}(\lambda)\right|_{\eta \rightarrow 0} & =I \otimes I+\eta r_{j, k}(\lambda)+O\left(\eta^{2}\right) \\
\left.G_{j}\right|_{\eta \rightarrow 0} & =I+\frac{\eta}{\Omega g}\left(1-\hat{N}_{j}\right)+O\left(\eta^{2}\right),
\end{aligned}
$$

here $r_{j, k}(\lambda)=\frac{P_{j, k}-1}{\lambda}$. Thus it follows that

$$
\left.t\left(\epsilon_{j}\right)\right|_{\eta \rightarrow 0}=1+\eta\left(\tau_{j}-\sum_{\substack{k=1 \\ k \neq j}}^{\Omega} \frac{1}{\epsilon_{j}-\epsilon_{k}}\right)+\cdots
$$

where

$$
\tau_{j}=\frac{1}{g}\left(1-\hat{N}_{j}\right)+\sum_{\substack{k=1 \\ k \neq j}}^{\Omega} \frac{\sum_{\alpha, \beta}^{2} E_{j}^{\alpha \beta} E_{k}^{\beta \alpha}}{\epsilon_{j}-\epsilon_{k}} .
$$

Here $E^{\alpha \beta}=|\alpha\rangle\langle\beta|, \alpha, \beta=1,2$ are the Hubbard operators. An immediate consequence from the Yang-Baxter algebra (2.6) is that $\left[\tau_{j}, \tau_{k}\right]=0$. Any Hamiltonian which is defined in terms of the mutually commuting set of operators $\left\{\tau_{j}, \hat{N}_{j}\right\}$ will necessarily be integrable where the operators in $(3.11)$ represent the constants of the motion. Explicitly,

$$
\begin{aligned}
H & =\sum_{j} \epsilon_{j} b_{j}^{+} b_{j}-g \sum_{j, k}\left(b_{j}^{+} b_{k}+b_{j} b_{k}^{+}\right) \\
& =-g \sum_{j=1}^{\Omega} \epsilon_{j} \tau_{j}+\sum_{j=1}^{\Omega} \epsilon_{j}+g^{3} \sum_{j, k}^{\Omega} \tau_{j} \tau_{k}-2 g \Omega+g\left(\sum_{j=1}^{\Omega} \hat{N}_{j}\right)^{2} .
\end{aligned}
$$

By employing the algebraic Bethe ansatz, the energy for the Hamiltonian $\left(\begin{array}{l}2 \\ 2\end{array}-1.1\right)$ is given by

$$
E=\sum_{j=1}^{M} v_{i}
$$


where the parameters $v_{i}$ satisfy the Bethe ansatz equation (Richardson's solution)

$$
\frac{1}{g}+\sum_{j=1}^{M} \frac{2}{v_{j}-v_{i}}=\sum_{n=1}^{\Omega} \frac{1}{2 \epsilon_{n}-v_{i}}
$$

Although complicated to solve it in general (see [3]1] for a numerical analysis), an asymptotic investigation of its solution can be performed. In the limit $g \rightarrow 0$ we can easily

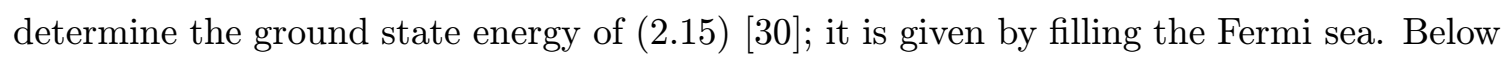
we will assume that the number of fermions is even. Thus for small $g>0$ it is appropriate to consider the asymptotic solution

$$
v_{i} \approx \epsilon_{i}+g \delta_{i}+g^{2} \mu_{i}, \quad i=1, \ldots, M
$$

Substituting this into (2.16it) and equating the different orders in $g$ gives us the asymptotic ground state energy

$$
E_{0} \approx 2 \sum_{j}^{m} \epsilon_{j}-g M+\frac{g^{2}}{2} \sum_{j=1}^{M} \sum_{k=M+1}^{\Lambda} \frac{1}{\epsilon_{j}-\epsilon_{k}} .
$$

Next we look at the first excited state. In the $g=0$ case this corresponds to breaking the Cooper pair at level $\epsilon_{M}$ and putting single unpaired electrons in the levels $\epsilon_{M}$ and $\epsilon_{M+1}$. Now these two levels become blocked. To solve the equations (2.16i) for this excited state is the same as for the ground state except that there are now $(M-1)$ Cooper pairs and we have to exclude the blocked levels. We can therefore write down the energy

$$
E_{1} \approx \epsilon_{M}+\epsilon_{M+1}+2 \sum_{j}^{M-1} \epsilon_{j}-g(M-1)+\frac{g^{2}}{2} \sum_{j=1}^{M-1} \sum_{k=M+2}^{\Omega} \frac{1}{\epsilon_{j}-\epsilon_{k}} .
$$

The gap is found to be

$$
\Delta \approx \epsilon_{M+1}-\epsilon_{M}+g+\frac{g^{2}}{2}\left(\sum_{j=1}^{M-1} \frac{1}{\epsilon_{M+1}-\epsilon_{j}}+\sum_{k=M+1}^{\Omega} \frac{1}{\epsilon_{k}-\epsilon_{M}}\right) .
$$

Observe that even though $g$ is assumed to be small, it may still be relatively larger than the level spacing of the single particle energies, which shows that the pairing interaction can still produce a gap in the asymptotic limit. Other important quantities, such as the correlation functions for zero temperature and the off-diagonal long range order parameter, can be computed in the asymptotic regime (see [1] $\left.\overline{6}_{1}^{1}, \overline{3} \overline{0}\right]$ for details). Having derived the reduced BCS model from an $R$-matrix associated with the $s u(2)$ algebra, it would be natural to investigate generalized BCS models constructed from $R$-matrices associated with Lie algebras. This is the content of the next section, where an integrable pairing model for two coupled BCS systems based on the su(4) algebra is constructed. 


\section{The two coupled BCS systems}

Now let us consider the following Hamiltonian [2] $\overline{2} \overline{9}]$

$$
\begin{aligned}
H= & B C S(1)+B C S(2)-g \sum_{j, k}^{\Omega} b_{j}^{+}(1) b_{j}^{+}(2) b_{k}(2) b_{k}(1) \\
& +g \sum_{j, k}^{\Omega} b_{j}^{+}(1) b_{k}(1)\left(n_{j}(2)-n_{k}(2)\right)^{2} \\
& +g \sum_{j, k}^{\Omega} b_{j}^{+}(2) b_{k}(2)\left(n_{j}(1)-n_{k}(1)\right)^{2}
\end{aligned}
$$

where

$$
B C S(a)=\sum_{j=1}^{\Omega} 2 \epsilon_{j} n_{j}(a)-g \sum_{j, k}^{\Omega} b_{j}^{+}(a) b_{k}(a) .
$$

Above the operators $b_{j}(a), b_{j}^{+}(a)$ are the annihilation and creation operators for the hardcore bosons (or Cooper pairs) in system $a$, and $j$ refers to the single particle energy level with energy $\epsilon_{j}$. We will assume that the values $\epsilon_{j}$ are distinct. Further, $g$ is a coupling strength constant for the scattering of Cooper pairs and $n_{j}(a)=b_{j}^{+}(a) b_{j}(a)$, is the Cooper pair number operator. As in the case of the usual BCS system there is a blocking effect (e.g. see $\left[\overline{1} \overline{1} \bar{j}_{1}\right)$, as there is no scattering of any unpaired states. For each level $j$ there are actually sixteen local states, but the nature of the Hamiltonian means that only on a subspace spanned by four of these states, where there are no unpaired states, is the scattering non-trivial (see $(\overline{3} . \overline{4})$ below). Hereafter we will restrict our analysis to this subspace.

On this restricted subspace the operators $b_{j}^{+}(a)=c_{j \uparrow}^{\dagger}(a) c_{j \downarrow}^{\dagger}(a), b_{j}(a)=c_{j \downarrow}(a) c_{j \uparrow}(a)$, where $c_{j \sigma}, c_{j \sigma}^{\dagger}, \sigma=\uparrow, \downarrow$, are the familiar fermion operators, satisfy the hard-core boson relations

$$
\begin{aligned}
& \left(b_{j}^{+}(a)\right)^{2}=0, \quad\left[b_{j}(a), b_{k}^{+}(b)\right]=\delta_{a b} \delta_{j k}\left(1-2 b_{j}^{+}(a) b_{j}(a)\right), \\
& {\left[b_{j}(a), b_{k}(b)\right]=\left[b_{j}^{+}(a), b_{k}^{+}(b)\right]=0, \quad \text { for } \mathrm{k} \neq \mathrm{j} .}
\end{aligned}
$$

We can see from the Hamiltonian expression that the exchange interaction of Cooper pairs in one system depends on the number of Cooper pairs in the other system. For example, if in system (1), the level $j$ is empty and the level $k$ is occupied by one Cooper pair, just for certain configurations of system (2) it is possible that this Cooper pair in (1) scatters

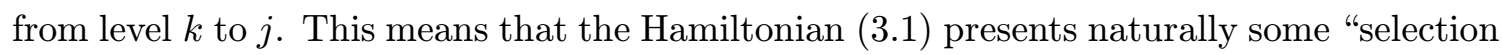
rules" for the scattering of states. We illustrate these configurations to indicate the possible 
pair scatterings

(1)

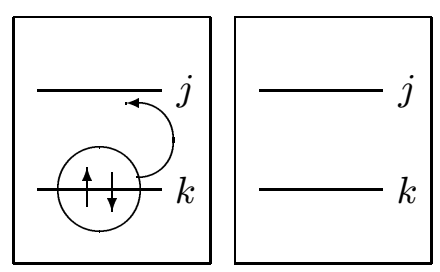

(1)

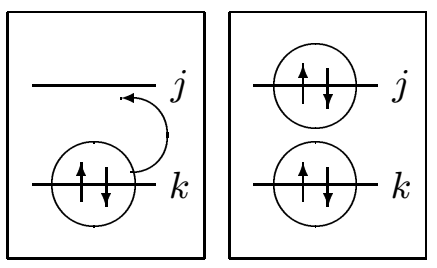

In addition, the double-pair scattering terms of the form

(1)

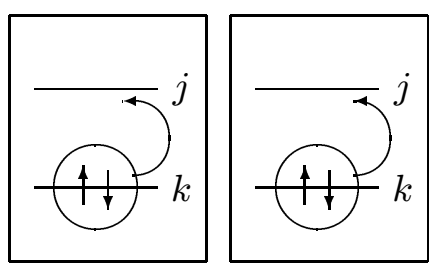

are also present. What the above indicates is that besides the number of Cooper pairs being conserved in each system, the number of double pairs (to be more precise, the number of energy levels which are completely filled) is also conserved. This can be seen in each of the scattering processes depicted graphically above. In each case the scattering does not overall change the number of completely filled levels. There are further symmetries in the Hamiltonian. For example, there is a reflection symmetry which interchanges the labels 1 and 2 for the two BCS systems. This arises as a result of a global $s o(3) \oplus u(1)$ symmetry that the model possesses, which will be made more clear later. In that which follows we shall first discuss the integrability of the Hamiltonian $\left(\overline{3} \cdot \bar{l}^{\prime}\right)$ in the context of the QISM.

Performing an analogous procedure as in the case before and taking into account that now we have the quantum $R$-matrix associated with the Lie algebra $s u(4)$, which acts in the tensor product of two 4-dimensional spaces $V \otimes V$ and can be written as

$$
R(\lambda)=\frac{(\lambda . I \otimes I+\eta P)}{(\lambda+\eta)}
$$

from which we can obtain the $s u(4)$ pairing Hamiltonian. For this purpose we make the following identification for the basis states

$$
|1\rangle=|0\rangle,|2\rangle=b^{+}(1) b^{+}(2)|0\rangle,|3\rangle=b^{+}(1)|0\rangle,|4\rangle=b^{+}(2)|0\rangle .
$$

and choose the G-matrix to be given by

$$
G \equiv \exp \left[\frac{2 \eta(1-n(1)-n(2))}{\Omega g}\right]=\left(\begin{array}{cccc}
\exp \left(\frac{2 \eta}{\Omega g}\right) & 0 & 0 & 0 \\
0 & \exp \left(\frac{-2 \eta}{\Omega g}\right) & 0 & 0 \\
0 & 0 & 1 & 0 \\
0 & 0 & 0 & 1
\end{array}\right)
$$


to construct the transfer matrix $t(\lambda)$. Thus it follows that

$$
\left.t\left(\epsilon_{j}\right)\right|_{\eta \rightarrow 0}=1+\eta\left(\tau_{j}-\sum_{\substack{k=1 \\ k \neq j}}^{\Omega} \frac{1}{\epsilon_{j}-\epsilon_{k}}\right)+\cdots
$$

where

$$
\tau_{j}=\frac{2}{g}\left(1-n_{j}(1)-n_{j}(2)\right)+\sum_{\substack{k=1 \\ k \neq j}}^{\Omega} \frac{\sum_{\alpha, \beta}^{4} E_{j}^{\alpha \beta} E_{k}^{\beta \alpha}}{\epsilon_{j}-\epsilon_{k}} .
$$

In addition, as a result of the $s o(3) \oplus u(1)$ symmetry mentioned earlier, it can be shown that there are extra conserved operators $K$ and $\chi$ such that

$$
\left[\tau_{j}, K\right]=\left[\tau_{j}, \chi\right]=[K, \chi]=0 .
$$

Above, $K$ is the Casimir operator of an $s o(3)$ subalgebra acting on the $\Omega$-fold tensor product

$$
K=\sum_{j, k}^{\Omega}\left(L_{j}^{+} L_{k}^{-}+L_{j}^{-} L_{k}^{+}+\frac{1}{2} L_{j}^{0} L_{k}^{0}\right)
$$

where $\left(L^{0}, L^{+}, L^{-}\right)$are the basis elements of this canonical so(3) subalgebra

$$
\begin{aligned}
& L^{+}=E^{34}=b^{+}(1) b(2), \\
& L^{-}=E^{43}=b^{+}(2) b(1), \\
& L^{0}=E^{33}-E^{44}=n(1)-n(2) .
\end{aligned}
$$

The $u(1)$ operator $\chi$ explicitly reads

$$
\begin{aligned}
\chi & =\sum_{j=1}^{\Omega}\left(E_{j}^{33}+E_{j}^{44}\right) \\
& =\sum_{j=1}^{\Omega}\left(n_{j}(1)-n_{j}(2)\right)^{2} .
\end{aligned}
$$

Any Hamiltonian which is defined in terms of the mutually commuting set of operators

$$
\left\{\tau_{j}, K, \chi\right\}
$$

will necessarily be integrable where the operators in (3.11) represent the constants of the motion. By making the following choice

$$
\begin{aligned}
H= & -g \sum_{j=1}^{\Omega} \epsilon_{j} \tau_{j}+\frac{g^{3}}{16} \sum_{j, k=1}^{\Omega} \tau_{j} \tau_{k}+\frac{3 g^{2}}{4} \sum_{j=1}^{\Omega} \tau_{j}+\frac{g}{2} K \\
& +\frac{g}{2} \chi(\chi-\Omega)+2 \sum_{j}^{\Omega} \epsilon_{j}+\frac{g \Omega^{2}}{4}-2 g \Omega
\end{aligned}
$$


we produce the Hamiltonian $(\overline{3} \cdot \overrightarrow{1} \cdot \overrightarrow{1})$.

Besides proving the integrability of the model, we can also obtain its exact solution from the algebraic Bethe ansatz for the standard su(4) vertex model constructed from the R-matrix ( $\left.\overline{3} \cdot \overline{3}_{i}\right)$. The eigenvalues of the integrals of motion $\tau_{j}(\overline{3} \cdot \bar{i})$ can be obtained from the expansion of the eigenvalue of the transfer matrix in the parameter $\eta$. Explicitly, the eigenvalues of $\tau_{j}$ are given by

$$
\Lambda_{j}=\frac{2}{g}+\sum_{l=1}^{N} \frac{1}{v_{l}-\epsilon_{j}}+\sum_{\substack{k=1 \\ k \neq j}}^{\Omega} \frac{1}{\epsilon_{j}-\epsilon_{k}},
$$

where the parameters satisfy the following equations

$$
\begin{aligned}
& \frac{4}{g}+\sum_{i=1}^{\Omega} \frac{1}{v_{j}-\epsilon_{i}}+\sum_{l=1}^{M} \frac{1}{v_{j}-u_{l}}=2 \sum_{\substack{l=1 \\
l \neq j}}^{N} \frac{1}{v_{j}-v_{l}}, \\
& \frac{2}{g}-\sum_{i=1}^{N} \frac{1}{u_{m}-v_{i}}-2 \sum_{\substack{l=1 \\
l \neq m}}^{M} \frac{1}{u_{l}-u_{m}}=\sum_{l=1}^{Q} \frac{1}{u_{m}-w_{l}}, \\
& \sum_{l=1}^{M} \frac{1}{w_{k}-u_{l}}=2 \sum_{\substack{l=1 \\
l \neq k}}^{Q} \frac{1}{w_{k}-w_{l}}, \\
& j=1, \cdots, N, \quad m=1, \cdots, M, \quad k=1, \cdots, Q
\end{aligned}
$$

We will also need the eigenvalues of the operators $K$ and $\chi$. Through use of $(3,10$, 3.1.

$$
\frac{1}{2}(M-2 Q)(M-2 Q+2) .
$$

Defining $N(a)=\sum_{j=1}^{\Omega} n_{j}(a)$, the quantum numbers $N, M$ and $Q$ are given by

$$
\begin{aligned}
N & =N(1)+N(2)-N(1) N(2), \\
M & =N(1)+N(2)-2 N(1) N(2), \\
Q & =N(2)-N(1) N(2) .
\end{aligned}
$$

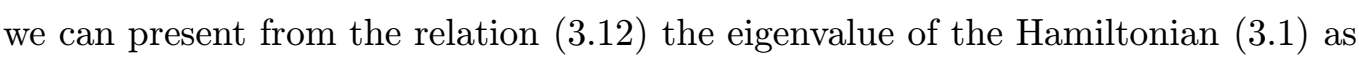

$$
E=4 \sum_{i=1}^{N} v_{i}-2 \sum_{m=1}^{M} u_{m}-g(2 N-3 M) .
$$

Let us make some small remarks about the degeneracies of the spectrum. Though the eigenstates of the Hamiltonian have not been made explicit here, it can be deduced by the standard arguments (e.g. [2] $\left.\left.\overline{6}_{i}^{i}\right]\right)$ that each is a highest weight state with respect to the $s o(3)$ symmetry algebra $\left(\overline{3}_{3}\right)$. In particular, the highest weight which is given by the eigenvalue 
of the operator $L^{0}$ is $M-2 Q$, so we can conclude that the multiplet generated by (3.9.9) acting on this highest weight state has dimension $M-2 Q+1$. Therefore for each solution of ( Since the Bethe ansatz equations take the form of coupled non-liner equations, it is very difficult to find analytical solutions. It is however possibly to conduct an asyptoic analysis for small value of the coupling parameters $g$, as previous model. An asyptotic analysis of the ground state, elementary excitations and the gap can be found in [i2 $2 \overline{9}]$.

\section{An extended BCS model}

The mechanism we presented above can be generalized to construct BCS pairing models associated with the Lie superalgebras. Let us consider a Hamiltonian associated with the Lie superalgebra $u(2 \mid 2)$, which reads

$$
\begin{aligned}
H= & \sum_{j=1}^{\Omega} \sum_{\sigma=+,-}\left(\epsilon_{j}-\mu_{0}-\sigma h\right) c_{j \sigma}^{\dagger} c_{j \sigma}+\mu_{e} \hat{N}_{e}+g \sum_{j, k}^{\Omega} c_{j+}^{\dagger} c_{j-}^{\dagger} c_{k-} \sigma_{k+} \\
& +g \sum_{j, k}^{\Omega} \sum_{\sigma=+,-} \tilde{c}_{j \sigma}^{\dagger} \tilde{c}_{k \sigma}-g \sum_{j, k}^{\Omega} \sum_{\sigma=+,-} c_{j \sigma}^{\dagger} c_{k \sigma} n_{j,-\sigma} n_{k,-\sigma},
\end{aligned}
$$

Above $g$ is the coupling constant, $j, k=1, \cdots, \Omega$ label a shell of doubly degenerate single electron energy level with energy $\epsilon_{j}$ and $n_{j \sigma}=c_{j \sigma}^{\dagger} c_{j \sigma}$ is the fermion number operator with spin $\sigma$ at the level $j$. Further $\hat{N}_{e}$ is the number of unpaired electrons denoted by $\hat{N}_{e}=\sum_{j=1}^{\Omega}\left(n_{j+}+n_{j-}-2 n_{j+} n_{j-}\right)$ and $\tilde{c}_{j \sigma}^{\dagger}=c_{j \sigma}^{\dagger}\left(1-n_{j,-\sigma}\right)$ denotes the confined electrons which prohibit the double-occupancy. The chemical potential is denoted by $\mu_{0}$ and $-\sigma h$ is the Zeeman energy of a spin $\sigma$ electron in a magnetic field. It is observed that both the total number of electrons $\hat{N}=\sum_{j=1}^{\Omega} \sum_{\sigma=+,-} n_{j \sigma}$ and unpaired electrons $\hat{N}_{e}$ are conserved, i.e. $[\hat{N}, H]=\left[\hat{N}_{e}, H\right]=0$. There are further symmetries in the Hamiltonian, e.g. the timereverse symmetry and global $s u(2) \oplus u(1)$ symmetry later. If the excited states involve a broken Cooper pair, breaking the $u(1)$ symmetry, the parameter $\mu_{e}$ significantly affects the gap for the grain with an even number of total electrons. In contrast to the standard

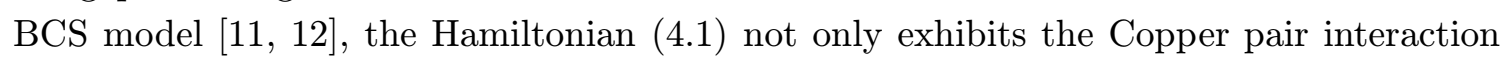
but also involves single electrons scattering. It is clear that the Hamiltonian (4.1) presents naturally configurations of possible electron scattering. It is reasonable that the excitation states consist of the mixture of the broken pairs, single electron scattering and Cooper pair scattering. The last term in the Hamiltonian (14.1) favors the the scattering between the Cooper pairs and single electrons, e.g. a Cooper pair at level $k$ could be possibly scattered to the level $j$ meanwhile the single electron at the level $j$ is scattered to the level $k$ ( see 
the second and fourth configurations in the Fig.(C) ).
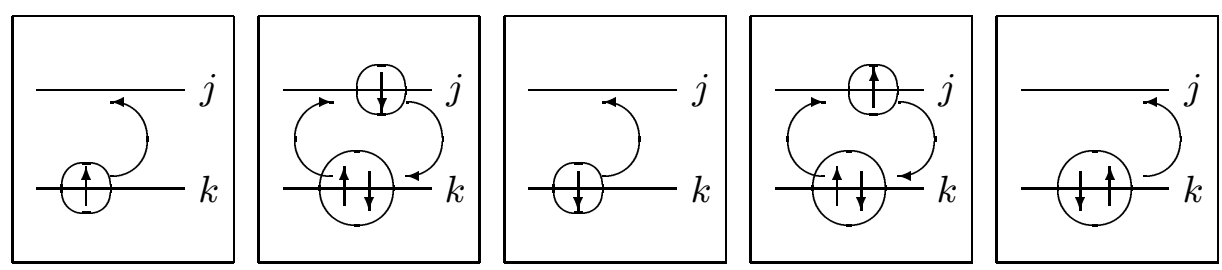

Fig. (C)

Although the Hamiltonian does not exhibit the blocking effect [1] involving the broken Cooper pairs and single electron scattering, the Pauli exclusion principle blocks a single electron and a Cooper pair to simutaneously occupy the same level. This effect weakens the pairing interaction. The Cooper pair atraction $(g<0)$ is believed to be signeficant in studying superconductivity for the ultrasmall metallic grains. If $g=0$, all Cooper pairs and single electrons occupy the lowest energy levels forming a Fermi sea.

Similarly to the previous sections, besides proving the integrability of the model (4i. we can also obtained its exact solution from the R-matrix associated with the $u(2 \mid 2)$ su-

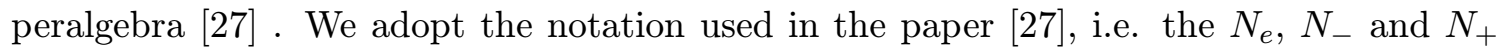
are the total number of unpaired electrons and the number of the unpaired electrons with spin - and + , respectively. The number of local electron pairs and the number of holes are denoted by $N_{l}$ and $N_{h}$, respectively. $N_{b}=N_{l}+N_{h}$ denote the total number of bosons.

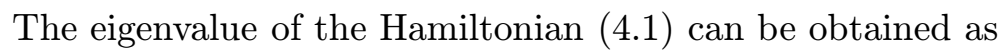

$$
E=2 \sum_{k=1}^{N_{e}+N_{l}} \lambda_{k}-\sum_{j=1}^{N_{e}} \lambda_{j}^{(1)}-\left(g+\mu_{0}\right)\left[N_{e}+2 N_{l}\right]+\mu_{e} N_{e}+h\left(N_{-}-N_{+}\right),
$$

where the parameters $\lambda, \lambda^{(1)}$ and $\lambda^{(2)}$ satisfy the following equations

$$
\begin{aligned}
& \frac{2}{g}-\sum_{i=1}^{\Omega} \frac{1}{\lambda_{j}-\epsilon_{i}}=\sum_{l=1}^{N_{e}} \frac{1}{\lambda_{j}-\lambda_{l}^{(1)}}+2 \sum_{\substack{l=1 \\
l \neq j}}^{N_{e}+N_{l}} \frac{1}{\lambda_{l}-\lambda_{j}}, \\
& -\frac{1}{g}+\sum_{k=1}^{N_{e}+N_{l}} \frac{1}{\lambda_{k}-\lambda_{m}^{(1)}}=\sum_{k=1}^{N_{+}} \frac{1}{\lambda_{k}^{(2)}-\lambda_{m}^{(1)}}, \\
& 2 \sum_{\substack{l=1 \\
l \neq n}}^{N_{+}} \frac{1}{\lambda_{l}^{(2)}-\lambda_{n}^{(2)}}+\sum_{j=1}^{N_{e}} \frac{1}{\lambda_{n}^{(2)}-\lambda_{j}^{(1)}}=0, \\
& j=1, \cdots, N_{e}+N_{l}, \quad m=1, \cdots, N_{e}, \quad n=1, \cdots, N_{+} .
\end{aligned}
$$

In principle, the Bethe ansatz results presented above can provide the exact solution for the ground state and all possible excitations. Nevertheless, the solutions of the Bethe equations are extremely complicated in the case $g \neq 0$. However, if we consider $|g|$ very small ( $g<0$, corresponding to the attraction interaction between the paired electrons), these equations can be analysed in an asymptotic way. These results will be considered elsewhere. 


\section{Conclusion}

To summarize, we have presented the construction of the integrable BCS pairing Hamiltonians based on the $s u(2), s u(4)$ Lie algebras. The first model corresponds to the standard reduced BCs Hamiltonian, while the second can be interpreted as describing two coupled BCS systems of different types, such as for protons and neutrons in a nuclear system. The Bethe ansatz equations and the energies for the models have been calculated. In addition, we have presented an extended BCS model of pairing correlations based on Lie superalgebra $u(2 \mid 2)$, which has the important property of describing also the scattering of single electrons besides of having the Cooper pair scattering. It is possible to extend this procedure to construct supersymmetric BCS models based on the $g l(m \mid n)$-Gaudin models

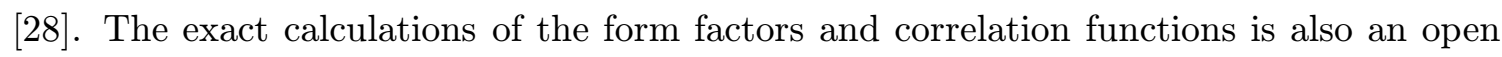
problem.

\section{Acknowledgments}

XWG would like to thank FAPERGS - Fundação de Amparo a Pesquisa do Estado do Rio Grande do Sul for financial support. AF thanks the organizers of the Workshop on Integrable Theories, Solitons and Duality for financial support.

\section{References}

[1] H Bethe, Z. Phys. 71 (1931) 205

[2] R. J. Baxter, Exactly Solved Models in Statistical Mechanics, Acad. Press, 1982.

[3] C. N. Yang, Phys. Rev. Lett. 19 (1967) 1312.

[4] E. H. Lieb and F. Y. Wu, Phys. Rev. Lett. 20 (1968) 1445.

[5] L. A. Takhtajan and L. D. Faddeev, Russian Mathem. Surveys 34 (1979) 11; E. Sklyanin, J. Sov. Mathem. 47 (1989) 2473.

[6] M.J. Martins and P.B. Ramos, Nucl. Phys. B 522 (1998) 413.

[7] X.-W. Guan, J. Phys. A: Math. Gen. 33 (2000) 5391.

[8] F.H.L. Essler and V.E. Korepin, Phys. Rev. B46 (1992) 9147.

[9] A. Foerster and M. Karowski, Phys. Rev. B46 (1992) 9234; Nucl. Phys. B396 (1993) 611.

[10] D.C. Ralph, C.T. Black and M. Tinkham, Phys. Rev. Lett. 76 (1996) 688; 78 (1997) 4087.

[11] J. Bardeen, L.N. Cooper and J.R. Schrieffer, Phys. Rev. 108 (1957) 1175.

[12] R.W. Richardson, Phys. Lett.3 (1963) 277; 5 (1963) 82;

R.W. Richardson and N. Sherman, Nucl. Phys. 52 (1964) 221; 52 (1964) 253.

[13] M.C. Cambiaggio, A.M.F. Rivas and M. Saraceno, Nucl. Phys. A 624 (1997) 157.

[14] G. Sierra, Nucl. Phys. B 572 (2000) 517.

[15] J. von Delft and D. C. Ralph, Phys. Rep. 345 (2001) 61;

J. von Delft, Ann. Phys. (Leipzig) 10 (2001) 219. 
[16] H.-Q. Zhou, J. Links, R.H. McKenzie and M.D. Gould, Phys. Rev. B 65 (2002) 060502(R).

[17] J. von Delft and R. Poghossian, cond-mat/0106405.

[18] H.M. Babujian, J. Phys. A: Math. Gen. 26 (1993) 6981;

H.M. Babujian and R. Flume, Mod. Phys. Lett. A9 (1994) 2029.

[19] E.K. Sklyanin, J. Sov. Math., 47 (1989) 2473; Lett. Math. Phys. 47 (1999) 275.

[20] N. Kitanine, J.M. Maillet, V. Terras, Nucl. Phys. B 554 (1999) 647;

J.M. Maillet and V. Terras, Nucl. Phys. B 575 (200) 627;

F. Göhmann and V.E. Korepin, J. Phys. A: Math. Gen. 33 (2000) 1199.

[21] L. Amico and A. Osterloh, Phys Rev Lett 88 (2002) 127003;

L. Amico, A.D. Lorenzo and A. Osterloh, Phys. Rev. Lett. 86 (2001) 5759; cond-mat/0105537.

[22] J. Dukelsky, C. Esebbag and P. Schuck, Phys. Rev. Lett. 87 (2001) 66403.

[23] M. Asorey, F. Falceto and G. Sierra, Nucl. Phys. B 622 (2002) 593.

[24] J. Links, H.-Q. Zhou, M.D. Gould and R.H. McKenzie, J. Phys. A : Math. Gen. 35 (2000) 6459.

[25] J. Links, H.-Q. Zhou, R.H. McKenzie and M.D. Gould, Int. J. Mod. Phys. B 16 3429; J. Links and K.E. Hibberd, Integrable coupling in a model for Josephson tunneling between non-identical BCS systems, Int. J. Mod. Phys. B 16 2009-2015.

[26] A.N. Kirillov, J. Sov. Math. 36 (1987) 115.

[27] F.H.L. Essler, V.E. Korepin and K. Schoutens, Phys. Rev. Lett. 68 (1992) 2960; F.H.L. Essler and V.E. Korepin, Phys. Rev. B46 (1992) 9147.

[28] X.-W. Guan, Z. Xiong, H.-Q. Zhou, Modern Physics Letters, A7 (1992) 1647.

[29] X.-W. Guan, A. Foerster, J. Links, H.-Q. Zhou, Nucl. Phys. B 642 (2002) 501.

[30] A. Foerster, J. Links and H.-Q. Zhou, Exact solvability in contemporary Physics, (2002), Review to appear in the "Special Issue on Integrable systems", Proceedings of the Indian National Science Academy.

[31] J.M. Roman, G. Sierra and J. Dukelsy Cond-matter/0202070. 